\title{
Editorial
}

\section{IFP ENERGIES NOUVELLES INTERNATIONAL CONFERENCE LES4ICE 2014 - LARGE-EDDY SIMULATION FOR INTERNAL COMBUSTION ENGINE FLOWS}

\author{
C. Angelberger ${ }^{1}$ and C. Mounaim-Rousselle ${ }^{2}$ \\ 1 IFP Energies nouvelles, 1-4 avenue de Bois-Préau, 92852 Rueil-Malmaison Cedex - France \\ 2 Laboratoire PRISME, Université d'Orléans, 8 rue Léonard de Vinci, 45072 Orléans Cedex 2 - France
}

Further improving the environmental performances of Internal Combustion Engines (ICE) increasingly requires moving beyond traditional multidimensional simulation tools based on a cycle averaged approach (Reynolds-Averaged Navier-Stokes, RANS), and to reliably predict and control individual engine cycles under realistic operating conditions. Large-Eddy Simulation (LES) offers this unique potential by predicting spatially filtered flow realizations, thus opening up new perspectives for extending the scope of application of Computational Fluid Dynamics (CFD) for ICE.

Since its $1^{\text {st }}$ edition in 2008, the LES4ICE conference provides a forum for exchange concerning research and development of LES and related experimental techniques for their application to ICE flows. It brings together researchers and engineers working in the field of piston engine combustion to debate the state of the art in LES applied to ICE and examine advanced experimental techniques capable of supporting and validating its development.

The present special issue proposes an extract from the contributions to the 2014 edition of LES4ICE, following a selection by the conference's Scientific Committee. The selected four articles provide a view on some recent advances in the domain of LES and related experimental techniques, and on their application to ICE flows.

The study of Cyclic Combustion Variability (CCV) in Spark-Ignition Engines (SIE) has become a prominent topic for LES research in the past decade. It has been demonstrated how combining advanced optical diagnostics and state-of-the-art LES can allow identifying the causes of CCV, and that the acquired understanding could efficiently be capitalized in the form of reduced models for 1D CFD simulations of complete engine systems. This on-going research opened exciting perspectives for the application of LES to other yet poorly understood and mastered non-cyclic phenomena in SIE, as in particular the link between CCV and engine knock.

A longstanding issue when studying the causes of CCV in SI engines is the relative importance of the variability of large-scale coherent structures, and of small scale turbulent fluctuations. A method aiming at addressing this question was proposed by S. Buhl, F. Hartmann and C. Hasse, in their contribution "Identification of Large-Scale Structure Fluctuations in IC Engines using POD-Based Conditional Averaging" [1]. They propose a method combining POD and conditional averaging, and discuss its application to the analysis of the origins of CCV for two different LES datasets. 
A key phenomenon for ensuring accurate predictions of SIE combustion is the reproduction of the flow generated during the intake phase. This is the topic addressed by two other papers that describe a combined use of optical diagnostics and LES. In their paper "Spatially Resolved Experimental and Numerical Investigation of the Flow through the Intake Port of an Internal Combustion Engine" [2], F. Hartmann, S. Buhl, F. Gleiss, P. Barth, M. Schild, S.A. Kaiser and C. Hasse present a detailed study of the impact of the flow in the valve seat region of the intake duct of a SI engine on the tumble flow generated inside the combustion chamber, using a combination of 2D2C PIV and Detached-Eddy Simulation. The article entitled "TCC-III Engine Benchmark for Large-Eddy Simulation of IC Engine Flows" [3] by P. Schiffmann, S. Gupta, D. Reuss, V. Sick, X. Yang and T.-W. Kuo presents recent evolutions of a simplified engine configuration used to acquire PIV data on the flow velocities during an engine stroke, and how they were used to compare the performances of two subgrid-scale closures. The impact of the numerical discretization of the intake valves is also illustrated.

The rationale for applying LES to Compression-Ignition Engines (CIE) is somewhat different. The complex interaction of flow, liquid spray, mixing and combustion found in CIE can lead to important cyclic, local variations of composition and temperature fields. This may have a very important impact on pollutant production that can hardly be addressed reliably using cycle averaged simulation tools. LES could contribute yielding a more reliable and quantitative prediction of CIE combustion and pollutant emissions. In this context, the paper entitled "Large-Eddy Simulation (LES) of Spray Transients: Start and End of Injection Phenomena" [4] contributed by M. Battistoni, Q. Xue and S. Som illustrates the usage of LES to study in a detailed manner highly transient phenomena during the start and end of injection of a Diesel spray under engine-like conditions, and compares LES predictions with findings from experiments based on $\mathrm{X}$-ray visualizations of these phases.

We hope the reader of this special issue will appreciate the proposed illustrations of how combining advanced optical diagnostics with cutting-edge LES could contribute designing and optimizing processes to allow a better understanding and mastering of non-cyclic and highly transient engine flow, spray, mixing and combustion phenomena.

\section{REFERENCES}

1 Buhl S., Hartmann F., Hasse C. (2016) Identification of Large-Scale Structure Fluctuations in IC Engines using POD-Based Conditional Averaging, Oil Gas Sci. Technol. 71, 1.

2 Hartmann F., Buhl S., Gleiss F., Barth P., Schild M., Kaiser S.A., Hasse C. (2016) Spatially Resolved Experimental and Numerical Investigation of the Flow through the Intake Port of an Internal Combustion Engine, Oil Gas Sci. Technol. 71, 2.

3 Schiffmann P., Gupta S., Reuss D., Sick V., Yang X., Kuo T.-W. (2016) TCC-III Engine Benchmark for Large-Eddy Simulation of IC Engine Flows, Oil Gas Sci. Technol. 71, 3.

4 Battistoni M., Xue Q., Som S. (2016) Large-Eddy Simulation (LES) of Spray Transients: Start and End of Injection Phenomena, Oil Gas Sci. Technol. 71, 4. 


\title{
Éditorial
}

\section{RENCONTRES SCIENTIFIQUES D'IFP ENERGIES NOUVELLES LES4ICE 2014 - LA SIMULATION AUX GRANDES ÉCHELLES POUR LES ÉCOULEMENTS DANS LES MOTEURS À COMBUSTION INTERNE}

\author{
C. Angelberger ${ }^{1}$ et C. Mounaim-Rousselle ${ }^{2}$ \\ 1 IFP Energies nouvelles, 1-4 avenue de Bois-Préau, 92852 Rueil-Malmaison Cedex - France \\ ${ }^{2}$ Laboratoire PRISME, Université d'Orléans, 8 rue Léonard de Vinci, 45072 Orléans Cedex 2 - France
}

L'amélioration des performances environnementales des Moteurs à Combustion Interne (MCI) requiert de façon croissante d'aller au-delà d'outils traditionnels de simulation multidimensionnels basés sur une approche de type cycle moyen (Reynolds-Averaged Navier-Stokes, RANS), pour permettre de prédire de manière fiable et pour contrôler des cycles moteur individuels pour des conditions opératoires réalistes. La simulation aux grandes échelles (Large-Eddy Simulation, LES) a ce potentiel unique du fait de sa capacité à prédire des réalisations physiques de cycles individuels filtrés spatialement, ouvrant ainsi de nouvelles perspectives pour étendre le champ d'application de la simulation numérique des MCI.

Depuis sa première édition en 2008, la conférence LES4ICE fournit un forum d'échange concernant la recherche et développement de la LES et de techniques expérimentales s'y rapportant, pour leur application à des écoulements moteur. Elle rassemble des chercheurs et ingénieurs actifs dans le domaine de la combustion moteur pour débattre de l'état de l'art, de l'application de la LES aux MCI, et pour étudier des techniques expérimentales avancées permettant d'appuyer son développement et de valider ses prédictions.

Le présent dossier OGST propose un aperçu des contributions faites à l'édition 2014 de LES4ICE, issu d'un processus de sélection par le Comité Scientifique de la conférence. Les quatre papiers ainsi retenus donnent une vue de progrès récents dans le domaine de la LES et de techniques expérimentales s'y rapportant, ainsi que concernant leur application à des écoulements moteur.

L'étude de Variations Cycliques de Combustion (VCC) dans les Moteurs à Allumage Commandé (MAC) est devenue un sujet prédominant de recherche en LES dans la dernière décennie. Il a ainsi été montré comment la combinaison de diagnostics optiques avancés et de l'état de l'art de la LES peut permettre d'identifier les causes des VCC, et que la compréhension ainsi acquise peut être capitalisée sous la forme de modèles réduits pour la simulation monodimensionnelle de systèmes moteur complets. Ce domaine de recherche d'actualité a ouvert d'intéressantes perspectives pour l'application de la LES à d'autres phénomènes acycliques encore mal compris et maitrisés dans les MAC, tels en particulier le lien entre VCC et cliquetis.

Une problématique de longue date concernant l'étude des origines des VCC dans les MAC est l'importance relative des variabilités des grandes échelles cohérentes et des fluctuations turbulentes aux petites échelles. Une méthode pour explorer cette question a été proposée par S. Buhl, 
F. Hartmann et C. Hasse dans leur contribution «Identification de fluctuations de structure aux grandes échelles dans les moteurs à combustion interne utilisant des moyennes conditionnelles basées sur la POD» [1]. Ils proposent une méthode combinant analyse POD et moyennes conditionnelles, et illustrent leur application à l'analyse des origines des VCC à l'aide de deux bases de données LES.

Un aspect essentiel pour permettre une prédiction fiable de la combustion dans les MAC est la reproduction de l'écoulement généré durant la phase d'admission. C'est la thématique explorée par deux autres articles qui rapportent une utilisation combinée de diagnostics optiques et de LES. Dans leur article "Étude numérique et expérimentale résolue dans l'espace de l'écoulement à travers le conduit d'admission d'un moteur à combustion interne » [2], F. Hartmann, S. Buhl, F. Gleiss, P. Barth, M. Schild, S.A. Kaiser et C. Hasse présentent une étude détaillée de l'impact de l'écoulement dans les sièges des soupapes d'admission d'un MAC sur l'écoulement de type tumble dans la chambre de combustion, en combinant PIV bidimensionnelles à deux composantes et une approche de type Detached-Eddy Simulation. L'article intitulé «Benchmark de moteur de référence TCC-III pour la simulation aux grandes échelles (Large-Eddy Simulations, LES) de l'écoulement dans les moteurs à combustion interne » [3] de P. Schiffmann, S. Gupta, D. Reuss, V. Sick, X. Yang et T.-W. Kuo présente des évolutions récentes réalisées sur une configuration monocylindre simplifiée utilisée pour acquérir des données PIV sur les champs de vitesse durant un cycle moteur, et comment elles furent exploitées pour comparer les performances de deux modèles de fermeture sous-maille. L'impact de la discrétisation numérique des soupapes d'admission est également illustré.

L'angle d'attaque pour l'application de la LES à l'étude de moteurs de type Diesel est quelque peu différent de celui pour les MAC. Les interactions complexes de l'écoulement, de jets liquides, du mélange et de la combustion dont les moteurs Diesel sont le siège, peuvent entrainer d'importantes variations locales cycliques des champs de température et de composition. Cela peut avoir comme conséquence une variabilité cyclique importante de la production de polluants, qui ne peut guère être adressée par des outils de simulation basés sur des cycles moyens. La LES pourrait contribuer à une prédiction plus fiable et quantitative de la combustion et des polluants de moteurs Diesel.

Dans ce contexte, l'article « Simulations aux grandes échelles (Large-Eddy Simulation, LES) de transitoires de jets liquides : phénomènes de début et fin d'injection » [4] de M. Battistoni, Q. Xue et S. Som montre l'utilisation de la LES pour étudier d'une manière détaillée les phénomènes fortement transitoires durant les phases de début et de fin d'injection d'un spray Diesel dans des conditions proches de celles dans un moteur, et compare les prédictions de la LES avec des résultats expérimentaux obtenus par visualisation par rayons $\mathrm{X}$ de ces phases.

Nous espérons que le lecteur de ce numéro spécial appréciera les illustrations qu'il propose du potentiel de la combinaison de diagnostics optiques avancés avec des LES de pointe pour permettre la conception et l'optimisation de méthodologies permettant une meilleure compréhension et maîtrise de phénomènes liés à l'écoulement, les sprays, le mélange et la combustion acycliques et fortement transitoires dans les moteurs.

\section{RÉFÉRENCES}

1 Buhl S., Hartmann F., Hasse C. (2016) Identification of Large-Scale Structure Fluctuations in IC Engines using POD-Based Conditional Averaging, Oil Gas Sci. Technol. 71, 1.

2 Hartmann F., Buhl S., Gleiss F., Barth P., Schild M., Kaiser S.A., Hasse C. (2016) Spatially Resolved Experimental and Numerical Investigation of the Flow through the Intake Port of an Internal Combustion Engine, Oil Gas Sci. Technol. 71, 2.

3 Schiffmann P., Gupta S., Reuss D., Sick V., Yang X., Kuo T.-W. (2016) TCC-III Engine Benchmark for Large-Eddy Simulation of IC Engine Flows, Oil Gas Sci. Technol. 71, 3.

4 Battistoni M., Xue Q., Som S. (2016) Large-Eddy Simulation (LES) of Spray Transients: Start and End of Injection Phenomena, Oil Gas Sci. Technol. 71, 4. 